\title{
ZETA-POLYNOMIALS, HILBERT POLYNOMIALS, AND THE EICHLER-SHIMURA IDENTITIES
}

\author{
MARIE JAMESON
}

\begin{abstract}
In 2017, Ono, Rolen, and Sprung [ORS17] answered problems of Manin [Man16] by defining zeta-polynomials $Z_{f}(s)$ for even weight newforms $f \in S_{k}\left(\Gamma_{0}(N)\right.$; these polynomials can be defined by applying the "Rodriguez-Villegas transform" to the period polynomial of $f$. It is known that these zeta-polynomials satisfy a functional equation $Z_{f}(s)= \pm Z_{f}(1-s)$ and they have a conjectural arithmetic-geometric interpretation. Here, we give analogous results for a slightly larger class of polynomials which are also defined using the Rodriguez-Villegas transform.
\end{abstract}

\section{Introduction ANd StATEMENT of Results}

Let $f \in S_{k}\left(\Gamma_{0}(N)\right.$ be a newform of even weight $k$ and level $N$, and let $L(f, s)$ be the $L$-function associated to $f$. Manin [Man16] speculated that the critical $L$-values

$$
L(f, 1), L(f, 2), \ldots, L(f, k-1)
$$

can be assembled in a natural way to build a zeta-polynomial. This polynomial $Z_{f}(s)$ should

(i) satisfy a functional-equation $Z_{f}(s)= \pm Z_{f}(1-s)$,

(ii) obey the "Riemann hypothesis:" if $Z_{f}(\rho)=0$ then $\operatorname{Re}(\rho)=1 / 2$, and

(iii) have an arithmetic-geometric interpretation.

Recently, Ono, Rolen, and Sprung ORS17 defined a zeta-polynomial $Z_{f}(s)$ which satisfies properties (i) and (ii) above. Assuming the BlochKato Tamagawa Number Conjecture, it also satisfies property (iii) by encoding the arithmetic of a combinatorial arithmetic-geometric object called BlochKato complex for $f$.

Although $Z_{f}(s)$ can be defined as a sum involving weighted moments of critical $L$-values and signed Stirling numbers of the first kind, it is more convenient here to instead express it in terms of (a slightly normalized version of) the period polynomial of $f$, which is given by

$$
R_{f}(X):=(\sqrt{N})^{k-1} \frac{(k-2) !}{(2 \pi)^{k-1}} \sum_{n=0}^{k-2} \frac{(2 \pi X)^{n}}{n !(\sqrt{N})^{n}} L(f, k-n-1) .
$$

Period polynomials are well-studied objects which are known to have many beautiful properties. For example, it is known that $R_{f}(X)$ satisfies its own Riemann hypothesis: all of its roots occur on the unit circle $|X|=1$, as proved in [EGR14, JMOS16]. See Section 2.1] 
for additional background information about $R_{f}(X)$. In [ORS17], $Z_{f}(s)$ is described as the unique polynomial which satisfies

$$
\frac{R_{f}(X)}{(1-X)^{w+1}}=\sum_{n \geq 0} Z_{f}(-n) X^{n},
$$

where $w:=k-2$. This relationship between $R_{f}(X)$ and $Z_{f}(s)$ is known as the "RodriguezVillegas transform," and a key theorem of Rodriguez-Villegas [RV02] allows Ono, Rolen, and Sprung to translate the Riemann hypothesis for $R_{f}(X)$ into statements (i) and (ii) about $Z_{f}(s)$.

Zeta-polynomials are relatively new objects, and little else is currently known about their properties. However, the results described thus far give evidence that known properties of a newform $f$ and its period polynomial $R_{f}(X)$ could be translated into the realm of the zetapolynomial $Z_{f}(s)$. This could give us more insight into the behavior of zeta-polynomials. The goal of this article is to offer additional evidence in this direction.

Note, however, that the results here are general enough that they do not require $R(X)$ to be the period polynomial of a newform (and for example, our results will apply to even/odd parts of period polynomials). Thus we fix the following notation: let $w \geq 2$ be even, let $R(X) \in \mathbb{C}[X]$ be any polynomial of degree at most $w$, and let $Z(s)$ be the unique polynomial which satisfies

$$
\frac{R(X)}{(1-X)^{w+1}}=\sum_{n \geq 0} Z(-n) X^{n},
$$

Our first result assumes the identity

$$
R(X)+\varepsilon i^{w} X^{w} R(1 / X)=0
$$

(where $\varepsilon= \pm 1$ is a constant) and interprets its meaning in terms of $Z(s)$. Note here that equation (2) is important because is it known to be true for any period polynomial (as well as its even and odd parts) associated to a newform $f \in S_{k}\left(\Gamma_{0}(N)\right)$, where $\varepsilon= \pm 1$ is the eigenvalue of $f$ under the Fricke involution.

Theorem 1. Let $w \geq 2$ be even, let $R(X) \in \mathbb{C}[X]$ be any polynomial of degree at most $w$, and let $Z(s)$ be the polynomial satisfying (1). If $R(X)$ satisfies (2), then we have that

$$
Z(s)+\varepsilon i^{w} Z(1-s)=0 .
$$

Remark 1. Since the period polynomial $R_{f}(X)$ satisfies equation (2) and the conclusion of the theorem gives property (i) above, one can view Theorem 1 as a generalization of property (i) proved by Ono, Rolen, and Sprung [ORS17] which uses completely different techniques (and does not depend on the Riemann hypothesis for period polynomials).

One may also consider what the "Eichler-Shimura relations" for period polynomials associated to cusp forms of level 1 (as described in Section 2) tell us about zeta-polynomials. Thus we suppose that

$$
\begin{aligned}
R(X)+(-i X)^{w} R(1 / X) & =0 \\
R(X)+(-i X)^{w} R\left(\frac{X-i}{-i X}\right)+(-i X-1)^{w} R\left(\frac{-i}{-i X-1}\right) & =0
\end{aligned}
$$


and obtain the following result.

Theorem 2. Let $w \geq 2$ be even, let $R(X) \in \mathbb{C}[X]$ be any polynomial of degree at most $w$, and let $Z(s)$ be the polynomial satisfying (11). If $R(X)$ satisfies (3) and (4), then

$$
Z(s)+i^{w} Z(1-s)=0
$$

and for any positive integer $n$ we have that

$$
\begin{aligned}
& Z(-n)+(-i)^{w} \sum_{m=1}^{n+1} a_{-m} Z(1-m)+ \\
& \quad \sum_{k \geq 0} \sum_{m=0}^{k+n} \sum_{j=0}^{k+n-m}\left(\begin{array}{c}
k+n \\
n
\end{array}\right)\left(\begin{array}{c}
m+w \\
w
\end{array}\right)\left(\begin{array}{c}
w+1 \\
j
\end{array}\right) \frac{(-1)^{j+1}(-i)^{k}}{(1-i)^{m+w+1}} Z(m+j-k-n)=0
\end{aligned}
$$

where

$$
\frac{(1-x)^{w+1}(x+i)^{n}}{(x+i-i x)^{w+1}(i x)^{n+1}}=\sum_{m=-n-1}^{\infty} a_{m} x^{m} .
$$

Example 1. For instance, consider the unique newform $\Delta \in S_{12}\left(\Gamma_{0}(1)\right)$. As computed in ORS17], we have that

$$
\begin{array}{r}
R_{\Delta}(X) \approx 0.114379 \cdot\left(\frac{36}{691} X^{10}+X^{8}+3 X^{6}+3 X^{4}+X^{2}+\frac{36}{691}\right) \\
+0.00926927 \cdot\left(4 X^{9}+25 X^{7}+42 X^{5}+25 X^{3}+4 X\right)
\end{array}
$$

and

$$
\begin{aligned}
& Z_{\Delta}(s) \approx\left(5.11 \times 10^{-7}\right) s^{10}-\left(2.554 \times 10^{-6}\right) s^{9}+\left(6.01 \times 10^{-5}\right) s^{8}-\left(2.25 \times 10^{-4}\right) s^{7} \\
& \quad+0.00180 s^{6}-0.00463 s^{5}+0.0155 s^{4}-0.0235 s^{3}+0.0310 s^{2}-0.0199 s+0.00596
\end{aligned}
$$

Since $\Delta$ is the unique normalized cusp form of weight 12 and level 1, we have that $R_{\Delta}(X)$ satisfies equations (2), (3), and (4), so Theorems 1 and 2 apply, although of course the first statement of Theorem 2 that

$$
Z_{\Delta}(s)-Z_{\Delta}(1-s)=0
$$

is the same as Theorem 1, and it is already known by [RS17.

However, one can now consider

$$
R_{\Delta}^{-}(X):=4 X^{9}+25 X^{7}+42 X^{5}+25 X^{3}+4 X
$$

(although we have abused notation a bit here by scaling to omit the constant 0.00926927). Although the roots of this polynomial can be understood using work of Conrey, Farmer, and Imamoglu in [CFI13, this polynomial does not satisfy the Riemann hypothesis that $R_{\Delta}(X)$ does, so the work in [ORS17] does not apply here. Since $R_{\Delta}^{-}(X)$ still satisfies equations (2),

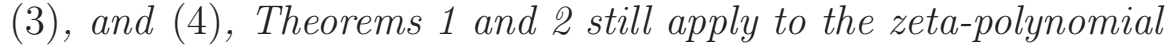

$$
\begin{array}{r}
Z_{\Delta}^{-}(s)=\frac{s^{10}}{36288}-\frac{5 s^{9}}{36288}+\frac{7 s^{8}}{2160}-\frac{367 s^{7}}{30240}+\frac{833 s^{6}}{8640}-\frac{2137 s^{5}}{8640} \\
+\frac{70841 s^{4}}{90720}-\frac{13193 s^{3}}{11340}+\frac{403 s^{2}}{360}-\frac{727 s}{1260} .
\end{array}
$$


In particular, we have that

$$
Z_{\Delta}^{-}(s)-Z_{\Delta}^{-}(1-s)=0 .
$$

Analogous statements hold for $Z_{\Delta}^{+}(s)$ as well.

Theorem 3. Let $w \geq 2$ be even, let $R(X) \in \mathbb{C}[X]$ be any polynomial of degree at most $w$, and let $Z(s)$ be the polynomial satisfying (11). If $R(X)$ satisfies (21) and $Z(s)$ has integer coefficients with positive leading term, then $Z(s)$ is a Hilbert polynomial.

This paper is organized as follows. In Section 2, we will review the relevant background related to period polynomials, zeta-polynomials, and Hilbert polynomials. In Sections 3, 4, and 5, we will prove Theorems 1, 2, and 3, respectively.

\section{Preliminaries}

2.1. Period polynomials of modular forms. First we must define our notation and review the required background related to modular forms and their period polynomials; period polynomials give a context for Theorems 1, 2, and 3 by providing natural applications of these results. For additional information, see, for example, the discussions in [KZ84] and [CPZ19.

Here we follow the standard notation: let $\mathbb{H}$ denote the upper half plane. For an even integer $k$ and $\gamma= \pm\left(\begin{array}{ll}a & b \\ c & d\end{array}\right) \in \mathrm{PGL}_{2}^{+}(\mathbb{R})$ we define the slash operator $\left.\right|_{k}$ for holomorphic functions $f: \mathbb{H} \rightarrow \mathbb{C}$ by

$$
\left(\left.f\right|_{k} \gamma\right)(\tau):=(a d-b c)^{k / 2}(c \tau+d)^{-k} f\left(\frac{a \tau+b}{c \tau+d}\right) .
$$

If $N$ is a positive integer, we let $S_{k}\left(\Gamma_{0}(N)\right)$ denote the space of cusp forms of weight $k$ on $\Gamma_{0}(N)$.

Now we summarize the theory of period polynomials. Let $f \in S_{k}\left(\Gamma_{0}(N)\right)$ be a cusp form of even weight $k$ and level $N$, and set $w:=k-2$. The period polynomial associated to $f$ is given by

$$
r_{f}(X):=\int_{0}^{\infty} f(\tau)(X-\tau)^{w} d \tau,
$$

which is a polynomial in the space

$$
\mathbb{V}_{w}:=\{P \in \mathbb{C}[X]: \operatorname{deg}(P) \leq w\} .
$$

We also define $r_{f}^{+}(X)$ and $r_{f}^{-}(X)$ to be the even and odd parts of $r_{f}(X)$, respectively, and note that $r_{f}^{ \pm}(X) \in \mathbb{V}_{w}^{ \pm}$(where of course $\mathbb{V}_{w}^{+}$and $\mathbb{V}_{w}^{-}$are defined to be the set of even and odd polynomials of degree at most $w$, respectively). There is an action of $\mathrm{PGL}_{2}^{+}(\mathbb{R})$ on $\mathbb{V}_{w}$ via the slash operator $\left.\right|_{-w}$.

Note that if the cusp form $f$ is an eigenfunction of the Fricke involution $W_{N}=\left(\begin{array}{cc}0 & -1 \\ N & 0\end{array}\right)$, i.e., $\left.f\right|_{k} W_{N}=\varepsilon f$ for $\varepsilon \in\{ \pm 1\}$, then it follows that $r_{f}$ (as well as $r_{f}^{ \pm}$) satisfies

$$
\left.r_{f}\right|_{w}\left(1+\varepsilon W_{N}\right)=0 .
$$


(This fact can also be obtained using the functional equation of the $L$-function associated to $f$.)

On the other hand, if the modular form $f$ has level $N=1$, then one can show that $r_{f}$ (as well as $r_{f}^{ \pm}$) satisfies the Eichler-Shimura relations

$$
\begin{array}{r}
r_{f} \mid(1+S)=0 \\
r_{f} \mid\left(1+U+U^{2}\right)=0,
\end{array}
$$

where

$$
S:=\left(\begin{array}{cc}
0 & -1 \\
1 & 0
\end{array}\right) \quad U:=\left(\begin{array}{cc}
1 & -1 \\
1 & 0
\end{array}\right)
$$

Thus we define

$$
\mathbb{W}_{w}:=\left\{P \in \mathbb{V}_{w}: P|(1+S)=P|\left(1+U+U^{2}\right)=0\right\}
$$

and note that $r_{f} \in \mathbb{W}_{w}$. The following result of Eichler-Shimura illustrates the importance of the period polynomial $r_{f}(X)$.

Theorem 4. The map

$$
\begin{aligned}
S_{k}\left(\mathrm{SL}_{2}(\mathbb{Z})\right) & \rightarrow \mathbb{W}_{w}^{-} \\
f & \mapsto r_{f}^{-}
\end{aligned}
$$

is an isomorphism. The map

$$
\begin{aligned}
S_{k}\left(\mathrm{SL}_{2}(\mathbb{Z})\right) & \rightarrow \mathbb{W}_{w}^{+} \\
f & \mapsto r_{f}^{+}
\end{aligned}
$$

is an injection whose image is a subspace of $\mathbb{W}_{w}^{+}$of codimension 1.

2.2. Zeta-polynomials for modular form periods. Let $f \in S_{k}\left(\Gamma_{0}(N)\right.$ be a newform of even weight $k \geq 4$. As discussed in the introduction, Ono, Rolen, and Sprung considered in ORS17] a reformulated version of the period polynomial

$$
R_{f}(X):=(\sqrt{N} / i)^{k-1} r_{f}(X / i \sqrt{N}) .
$$

These polynomials serve as the inspiration for this work, so we note here that equation (5) gives

$$
R_{f}(X)+\varepsilon i^{w} X^{w} R_{f}(1 / X)=0,
$$

i.e., $R_{f}(X)$ satisfies equation (2). Also, in the special case where $N=1$, the Eichler-Shimura relations (6) and (7) above tell us that

$$
\begin{aligned}
R_{f}(X)+(-i X)^{w} R_{f}(1 / X) & =0 \\
R_{f}(X)+(-i X)^{w} R_{f}\left(\frac{X-i}{-i X}\right)+(-i X-1)^{w} R_{f}\left(\frac{-i}{-i X-1}\right) & =0
\end{aligned}
$$

i.e., $R_{f}(x)$ satisfies equations (3) and (44) when $N=1$ (with $\varepsilon=1$ ). As discussed in the introduction, the zeta-polynomials for modular form periods $Z_{f}(s)$ are given by

$$
\frac{R_{f}(X)}{(1-X)^{w+1}}=\sum_{n \geq 0} Z_{f}(-n) X^{n} .
$$


2.3. Hilbert polynomials. Here we give the necessary background related to Hilbert polynomials; for more information, see [Bre98]. Fix a field $k$, let $R=\bigoplus_{j \geq 0} R_{j}$ be a graded $k$ algebra, and suppose that $R$ is standard (i,.e., that it can be finitely generated by elements of $\left.R_{1}\right)$. The Hilbert series of $R$ is the formal power series

$$
\sum_{j \geq 0} \operatorname{dim}_{k}\left(R_{j}\right) X^{j}
$$

It is known that the Hilbert series can be written as

$$
\frac{U(X)}{(1-X)^{r}}=\sum_{j \geq 0} \operatorname{dim}_{k}\left(R_{j}\right) X^{j}
$$

for some positive integer $r$ and some polynomial $U(X)$, and it is also known that there exists a polynomial $P_{R}(X) \in \mathbb{Q}[X]$ such that

$$
P_{R}(j)=\operatorname{dim}_{k}\left(R_{j}\right)
$$

for all sufficiently large $j$.

Thus we make the following definition: a polynomial $H(X) \in \mathbb{Q}[X]$ is called a Hilbert polynomial if there exists a standard graded $k$-algebra $R$ such that $H(X)=P_{R}(X)$. Work of Brenti [Bre98] investigates which polynomials are Hilbert polynomials, and how to measure "how far" a polynomial is from being Hilbert. Along the way, Brenti proves the following useful results.

Theorem 5 (Theorems 3.5 and 3.14 of [Bre98]). Let $H(X) \in \mathbb{Z}[X]$ be a polynomial with positive leading term.

- There exists $M \in \mathbb{N}$ such that $H(X+j)$ is a Hilbert polynomial for any $j \geq M$.

- If $H(X)$ is a Hilbert polynomial then $H(X+1)$ is a Hilbert polynomial.

\section{Proof of Theorem 1}

Let $w \geq 2$ be even, let $R(X)=\sum_{j=0}^{w} a_{j} X^{j} \in \mathbb{C}[X]$, and let $Z(s)$ be the polynomial satisfying

$$
\frac{R(X)}{(1-X)^{w+1}}=\sum_{n \geq 0} Z(-n) X^{n} .
$$

In order to better understand the relationship between $R(X)$ and $Z(s)$, we use Newton's Binomial Theorem, which says that

$$
\frac{1}{(1-X)^{w+1}}=\sum_{n \geq 0}\left(\begin{array}{c}
w+n \\
n
\end{array}\right) X^{n} .
$$


Thus we have

$$
\begin{aligned}
\sum_{n \geq 0} Z(-n) X^{n} & =\frac{R(X)}{(1-X)^{w+1}} \\
& =\left(\sum_{j=0}^{w} a_{j} X^{j}\right)\left(\sum_{n \geq 0}\left(\begin{array}{c}
w+n \\
n
\end{array}\right) X^{n}\right) \\
& =\sum_{n \geq 0}\left(\sum_{j=0}^{w} a_{j}\left(\begin{array}{c}
w+n-j \\
w
\end{array}\right)\right) X^{n}
\end{aligned}
$$

so we can now express $Z(s)$ explicitly by

$$
Z(s)=\sum_{j=0}^{w} a_{j}\left(\begin{array}{c}
w-s-j \\
w
\end{array}\right) .
$$

Now, to prove Theorem 1, we suppose equation (2), i.e., that

$$
a_{j}+\varepsilon i^{w} a_{w-j}=0
$$

for $0 \leq j \leq w$. Thus

$$
\begin{aligned}
Z(1-s) & =\sum_{j=0}^{w} a_{j}\left(\begin{array}{c}
w-1+s-j \\
w
\end{array}\right)=\sum_{j=0}^{w} a_{w-j}\left(\begin{array}{c}
w-1+s-(w-j) \\
w
\end{array}\right) \\
& =\sum_{j=0}^{w}-\varepsilon i^{w} a_{j}\left(\begin{array}{c}
-1+s+j \\
w
\end{array}\right)=\sum_{j=0}^{w}-\varepsilon i^{w} a_{j}\left(\begin{array}{c}
w-s-j \\
w
\end{array}\right) \\
& =-\varepsilon i^{w} Z(s),
\end{aligned}
$$

as desired.

\section{Proof of Theorem 2}

First, note that the first statement of Theorem 2 follows from Theorem 1 by letting $\varepsilon=1$. To complete the proof of Theorem 2, we note that equation (4) tells us that for any positive integer $n$, we have that

$$
\begin{aligned}
\frac{1}{2 \pi i} \int_{\gamma} \frac{R(z)}{(1-z)^{w+1}} & z^{-(n+1)} d z+\frac{1}{2 \pi i} \int_{\gamma} \frac{(-i z)^{w} R\left(\frac{z-i}{-i z}\right)}{(1-z)^{w+1}} z^{-(n+1)} d z \\
& +\frac{1}{2 \pi i} \int_{\gamma} \frac{(-i z-1)^{w} R\left(\frac{-i}{-i z-1}\right)}{(1-z)^{w+1}} z^{-(n+1)} d z=0
\end{aligned}
$$

where $\gamma$ is a small circle with center 0 (oriented counter-clockwise). Our proof will follow by interpreting each integral of equation (8) ; note that by Cauchy's integral formula, the first integral is

$$
\frac{1}{2 \pi i} \int_{\gamma} \frac{R(z)}{(1-z)^{w+1}} z^{-(n+1)} d z=Z(-n)
$$


Next, we note that the second integral is (by applying equation (3) and then setting $x=-i z /(z-i))$

$$
\begin{aligned}
\frac{1}{2 \pi i} \int_{\gamma} \frac{(-i z)^{w} R\left(\frac{z-i}{-i z}\right)}{(1-z)^{w+1}} z^{-(n+1)} d z & =\frac{-1}{2 \pi i} \int_{\gamma} \frac{(-i z)^{w}\left(\frac{z-i}{z}\right)^{w} R\left(\frac{-i z}{z-i}\right)}{(1-z)^{w+1}} z^{-(n+1)} d z \\
& =\frac{-(-i)^{w}}{2 \pi i} \int_{\gamma} \frac{R\left(\frac{-i z}{z-i}\right)(z-i)^{w+2}}{(1-z)^{w+1}} z^{-(n+1)} \frac{d z}{(z-i)^{2}} \\
& =\frac{(-i)^{w}}{2 \pi i} \int_{\gamma_{0}} \frac{R(x)}{(1-x)^{w+1}} \frac{(1-x)^{w+1}(x+i)^{w+1}(x+i)^{n+1}}{(x+i-i x)^{w+1}(x+i)^{w+2}(i x)^{n+1}} d x \\
& =\frac{(-i)^{w}}{2 \pi i} \int_{\gamma_{0}} \frac{R(x)}{(1-x)^{w+1}} \frac{(1-x)^{w+1}(x+i)^{n}}{(x+i-i x)^{w+1}(i x)^{n+1}} d x \\
& =(-i)^{w} \sum_{m=1}^{n+1} a_{-m} Z(1-m)
\end{aligned}
$$

where $\gamma_{0}$ is a small circle with center 0 and

$$
\frac{(1-x)^{w+1}(x+i)^{n}}{(x+i-i x)^{w+1}(i x)^{n+1}}=\sum_{m=-n-1}^{\infty} a_{m} x^{m} .
$$

Finally, by Cauchy's integral formula and equation (3), the third integral is the coefficient of $z^{n}$ in the expansion for

$$
\frac{(-i z-1)^{w} R\left(\frac{-i}{-i z-1}\right)}{(1-z)^{w+1}}=\frac{-R(z-i)}{(1-z)^{w+1}}
$$

about 0 . Thus we define a function $g$ by

$$
g(z-i)=\frac{-R(z-i)}{(1-z)^{w+1}}=\frac{-R(z-i)}{(1-z+i)^{w+1}} \cdot \frac{(1-z+i)^{w+1}}{(1-z)^{w+1}}
$$

Then

$$
\begin{aligned}
g(z) & =\frac{-R(z)}{(1-z)^{w+1}} \cdot \frac{(1-z)^{w+1}}{(1-i-z)^{w+1}} \\
& =\left(-\sum_{n \geq 0} Z(-n) z^{n}\right)(1-z)^{w+1}\left(\sum_{m \geq 0}\left(\begin{array}{c}
m+w \\
w
\end{array}\right) \frac{z^{m}}{(1-i)^{m+w+1}}\right) \\
& =\sum_{k \geq 0}\left[\sum_{m=0}^{k}\left(\begin{array}{c}
m+w \\
w
\end{array}\right) \frac{1}{(1-i)^{m+w+1}} \sum_{j=0}^{k-m}\left(\begin{array}{c}
w+1 \\
j
\end{array}\right)(-1)^{j+1} Z(m+j-k)\right] z^{k} .
\end{aligned}
$$


Now, set $b_{k}$ to be the expression inside the brackets above, so that $g(z)=\sum_{k \geq 0} b_{k} z^{k}$. Since the radius of convergence of this series is $\sqrt{2}$, we may substitute to find

$$
\begin{aligned}
g(z-i) & =\sum_{k \geq 0} b_{k}(z-i)^{k}=\sum_{n \geq 0} \sum_{k \geq 0} b_{k+n}\left(\begin{array}{c}
k+n \\
n
\end{array}\right)(-i)^{k} z^{n} \\
& =\sum_{n \geq 0}\left[\sum_{k \geq 0} \sum_{m=0}^{k+n} \sum_{j=0}^{k+n-m}\left(\begin{array}{c}
k+n \\
n
\end{array}\right)\left(\begin{array}{c}
m+w \\
w
\end{array}\right)\left(\begin{array}{c}
w+1 \\
j
\end{array}\right) \frac{(-1)^{j+1}(-i)^{k}}{(1-i)^{m+w+1}} Z(m+j-k-n)\right] z^{n} .
\end{aligned}
$$

Thus the third integral is $\sum_{k \geq 0} \sum_{m=0}^{k+n} \sum_{j=0}^{k+n-m}\left(\begin{array}{c}k+n \\ n\end{array}\right)\left(\begin{array}{c}m+w \\ w\end{array}\right)\left(\begin{array}{c}w+1 \\ j\end{array}\right) \frac{(-1)^{j+1}(-i)^{k}}{(1-i)^{m+w+1}} Z(m+j-k-n)$, completing the proof.

\section{Proof of Theorem 3}

Suppose for the sake of contradiction that $Z(s)$ is not a Hilbert polynomial. By the first part of Theorem 5, there exists some $M \in \mathbb{N}$ such that $Z(s+j)$ is Hilbert for any $j \geq M$ (and we may suppose without loss of generality that $M$ is minimal, i.e., that $Z(s+M)$ is Hilbert and $Z(s+M-1)$ is not Hilbert).

Note that by Theorem 1

$$
Z(s+M)=-\varepsilon i^{w} Z(1-M-s)
$$

is Hilbert, so the second part of Theorem 5 tells us that

$$
-\varepsilon i^{w} Z(2-M-s)=Z(M+s-1)
$$

is also Hilbert. This is a contradiction.

\section{REFERENCES}

[Bre98] Francesco Brenti. Hilbert polynomials in combinatorics. J. Algebraic Combin., 7(2):127-156, 1998.

[CFI13] John Brian Conrey, David W. Farmer, and Özlem Imamoglu. The nontrivial zeros of period polynomials of modular forms Lie on the unit circle. Int. Math. Res. Not. IMRN, (20):4758-4771, 2013.

[CPZ19] YoungJu Choie, Yoon Kyung Park, and Don B. Zagier. Periods of modular forms on $\gamma_{0}(n)$ and products of jacobi theta functions. J. Eur. Math. Soc. (JEMS), 21(5):1379-1420, 2019.

[EGR14] Ahmad El-Guindy and Wissam Raji. Unimodularity of zeros of period polynomials of Hecke eigenforms. Bull. Lond. Math. Soc., 46(3):528-536, 2014.

[JMOS16] Seokho Jin, Wenjun Ma, Ken Ono, and Kannan Soundararajan. Riemann hypothesis for period polynomials of modular forms. Proc. Natl. Acad. Sci. USA, 113(10):2603-2608, 2016.

[KZ84] W. Kohnen and D. Zagier. Modular forms with rational periods. In Modular forms (Durham, 1983), Ellis Horwood Ser. Math. Appl.: Statist. Oper. Res., pages 197-249. Horwood, Chichester, 1984.

[Man16] Yu. I. Manin. Local zeta factors and geometries under Spec Z. Izv. Ross. Akad. Nauk Ser. Mat., 80(4):123-130, 2016. Reprinted in Izv. Math. 80 (2016), no. 4, 751-758.

[ORS17] Ken Ono, Larry Rolen, and Florian Sprung. Zeta-polynomials for modular form periods. Adv. Math., 306:328-343, 2017.

[RV02] Fernando Rodriguez-Villegas. On the zeros of certain polynomials. Proc. Amer. Math. Soc., 130(8):2251-2254, 2002. 